\title{
POLÍTICA PÚBLICA Y TRABAJO SOCIAL: EL IMPACTO DEL PROCESO LEGISLATIVO Y SUS IMPLICACIONES PARA LA PROFESIÓN
}

\author{
Dennis Soto Fantauzzi ${ }^{1}$
}

\section{Resumen}

El trabajo social como profesión, enfrenta uno de sus más grandes retos al comienzo de un nuevo siglo: insertarse en el proceso de proponer e influenciar políticas públicas que ofrezcan soluciones noveles a las desigualdades sociales, la criminalización de la drogodependencia y el discrimen por condición social. El presente análisis les provee a los profesionales del trabajo social las estrategias necesarias para la integración del acervo de experiencias y conocimientos de la profesión en la formulación de políticas públicas, utilizando una perspectiva axiológica, tanto por la relevancia de su peritaje como de su experiencia de campo. A través de un análisis de las fases del proceso legislativo y las estrategias disponibles para la integración de los trabajadores sociales en la gestión pública y comunitaria, se analizarán algunas de las medidas legislativas que se encuentran ante la consideración de la Asamblea Legislativa de Puerto Rico, que afectan a los sectores vulnerables de nuestra sociedad.

Descriptores: política pública, trabajo social, perspectiva axiológica, proceso legislativo. 


\section{Abstract}

At the beginning of a new millennium, the discipline of social work faces a major challenge: to become an active participant in the endeavor of proposing and influencing the formulation of public policies which may promise novel solutions to inequality, crime, drug dependency and discrimination because of social status. The present analysis provides social workers with needed strategies for the employment of their experiences and knowledge into the formulation of social policies, through an axiological perspective that benefits the process with of the expertise and experience of the profession. Throughout the analysis of the various stages of the legislative process and the strategies available for the inclusion of social workers into the discussion of public and community affairs, we will examine some legislative projects considered by the Legislature of Puerto Rico that may benefit vulnerable people in the society.

Keywords: public policy, social work, axiological perspective, legislative process.

\section{Introducción}

Uno de los grandes retos que enfrenta el Trabajo Social como profesión al comienzo de un nuevo siglo, es la necesidad de identificar soluciones noveles a los problemas que históricamente han afectado a los sectores desprotegidos de la sociedad puertorriqueña ya que los problemas sociales que enfrenta nuestra sociedad están arraigados a factores complejos, multidimensionales y multicausales, estos requieren la intervención de profesionales competentes, así como de políticas públicas efectivas que contemplen la infraestructura, los recursos y los protocolos necesarios para atenderlas. Precisamente, el presente análisis aporta al entendimiento de la necesidad de integrar el trabajo social en el proceso ordinario para la formulación de políticas públicas y sociales.

Política social puede ser definida como el conjunto de actividades y principios de una sociedad que guían la manera en que ésta interviene y regula las relaciones entre los individuos, los grupos, las 
comunidades y las instituciones sociales. En consecuencia, la política social abarca el universo gubernamental, incluyendo los programas de educación, salud, prevención del crimen, corrección, seguridad económica y bienestar social, entre otros (Ruiz, 1997).

Como resultado de la naturaleza y la finalidad del trabajo social, esta profesión está llamada a contribuir con la formulación de una política social eficaz. Según Ruiz (1997), esta aportación se puede hacer desde tres vertientes esenciales. Primero, señalando y llamando la atención hacia las necesidades y problemas que requieren atención en el área de bienestar social. Segundo, analizando la política de bienestar social existente con miras a determinar los logros obtenidos, las limitaciones existentes y ofrecer recomendaciones sobre cambios a ser propuestos. Tercero, participando directamente en la elaboración de las políticas sociales. Esta misma autora advierte que al examinar el trasfondo histórico de la participación de profesionales del trabajo social en estos procesos, se hace evidente que la actividad relacionada con la formulación de la política social no ha sido tradicionalmente una de las favoritas de la profesión. En consecuencia, las políticas públicas concebidas e implantadas en el país, no se benefician, como debería ser, del acervo de experiencias y conocimientos de la profesión.

La literatura ha sido consistente en criticar el rol limitado que han jugado los y las trabajadores sociales en la actividad básica de desarrollar iniciativas de política social, empero a reconocer la importancia de tal inserción. Por ejemplo, Guardiola (1997), llevó a cabo un estudio utilizando una muestra probabilística de tipo aleatorio compuesta por 37 legisladores, de los cuales veinticinco (25) eran representantes y doce (12) eran senadores. Entre los hallazgos más relevantes, un $50 \%$ de los participantes informó que el peritaje de los trabajadores sociales ha estado ausente de su gestión legislativa; un $40 \%$ responsabilizó al limitado conocimiento de los trabajadores sociales sobre el proceso legislativo como la principal barrera en la interacción entre ambos y un 34\% evaluó como deficiente la participación de estos profesionales en el proceso de formulación de políticas públicas y sociales. Sin embargo, tal como establece Patti y Dear (según citado por Guardiola, 1997), los trabajadores sociales 
deben y pueden tener una influencia decisiva sobre el desarrollo de la política legislativa, particularmente en jugar un rol crucial en generar políticas sociales y en afectar el curso de la legislación, para que ésta sea más responsiva y pertinente.

\section{El Proceso de Influenciar: Una Introducción al Proceso Legislativo en Puerto Rico}

El proceso legislativo viabiliza que una iniciativa se convierta en ley, a través de de cinco fases fundamentales. En la primera fase, el legislador radica un proyecto de ley, por iniciativa propia o por petición, con la expectativa de que el mismo sea considerado por la Asamblea Legislativa. Una vez el proyecto es radicado, se asigna a una Comisión, la que determina si se inician vistas públicas a fin de auscultar los méritos del mismo. En este proceso, se examinan las circunstancias específicas que motivaron la radicación de la medida, por lo que se invita a personas o entidades interesadas en expresarse a favor o en contra. Una vez culminado este trámite, la Comisión emite un Informe en el que recomienda la aprobación o la no aprobación de la medida investigada. Si el Informe de la Comisión es favorable, el proyecto pasa a una tercera fase, en el que sus defectos y virtudes son analizados y debatidos en el Hemiciclo del cuerpo de origen (Soto, noviembre 2006).

Luego de completar el proceso de aprobación, el proyecto pasa a la consideración de la otra Cámara Legislativa (Cámara de Representantes o Senado de Puerto Rico, según sea el caso), la que repite todo el proceso, pero comenzando en la segunda fase. Una vez que el proyecto ha sido debidamente aprobado por ambas Cámaras, el mismo pasa a la consideración del Gobernador. Durante esta cuarta fase, el Gobernador impartirá su firma para que el proyecto se convierta en Ley o lo rechazará a través de los mecanismos del veto expreso o veto de bolsillo. El veto expreso es uno explícito en el que se ofrece una explicación sobre las reservas que tiene el Ejecutivo para no endosarlo. El veto de bolsillo ocurre cuando el Gobernador no firma el proyecto dentro del tiempo prescripto. Ante esta situación, la única forma en que un proyecto podría convertirse en Ley, a pesar 
de la objeción del Ejecutivo, sería que el mismo regresara a la Legislatura y dos terceras partes de los miembros de cada Cuerpo votaran para ir por encima del veto. La quinta fase corresponde a la promulgación realizada por el Secretario de Estado, quien informa a la ciudadanía sobre la aprobación de la nueva Ley (Soto, noviembre 2006).

Son muchos los proyectos de política social que pasan por este proceso. Durante los primeros dos años de la Decimoquinta Asamblea Legislativa, la cual comprende el cuatrienio 2005-2008, un total de 5,298 proyectos de ley han sido presentados, de los cuales una cantidad considerable son relevantes para el quehacer del trabajador social. Para demostrar la manera en que la inserción activa de profesionales en trabajo social puede beneficiar el proceso legislativo para formular política social, hemos seleccionado cuatro proyectos de ley dentro del universo de medidas que impactan a la profesión. Esta es una pequeña muestra de la gran variedad de proyectos relacionados a este tema sobre los cuales ha actuado la Asamblea Legislativa recientemente. Entendemos que los mismos ameritan una profunda reflexión debido a sus implicaciones para la profesión, luego de un proceso en el que la comunidad de trabajadores sociales estuvo ausente. Los proyectos a los que haré referencia son: el intento fallido para establecer la carta de derechos de las personas sin hogar (P. de la C. 1454); la prohibición de discrimen por condición social (P. del S. 223); la prohibición de discrimen por impedimento físico y orientación sexual (P. del S. 1585) y la solicitud de investigación para auscultar la viabililidad de reglamentar la prostitución y decriminalizar la adicción en Puerto Rico (R. del S. 2426).

\section{La Carta de Derechos de las Personas sin Hogar: Una Agenda Inconclusa}

Desde el año 2001, un proyecto de ley que establecería "La Carta de Derechos de la Persona sin Hogar del Estado Libre Asociado de Puerto Rico" no ha encontrado el respaldo necesario para ser convertido en Ley. Esta iniciativa fue trabajada durante el presente cuatrienio (2004- 
2008), a través del Proyecto de la Cámara 1454. El proyecto estaba dirigido a garantizar el derecho de las personas sin hogar a recibir un albergue adecuado, servicios nutricionales, atención médica en su fase preventiva, clínica y de rehabilitación, facilitar un plan remediativo para reingresar a la fuerza laboral, protección contra cualquier tipo de maltrato o abuso y el derecho al libre acceso a las plazas, parques y demás facilidades públicas, entre otros. Mientras las personas con impedimentos, de edad avanzada, los niños y adultos con síndrome de autismo, las estudiantes embarazadas, los portadores del virus VIH, los pacientes de salud mental y los veteranos, entre otros, cuentan con una carta de derechos que les cobija bajo el principio de equidad que persigue nuestro ordenamiento jurídico, las personas sin hogar se encuentran desprovista en la protección de sus derechos fundamentales.

Luego de concluir el proceso de análisis, las Comisiones de Bienestar Social y Vivienda_de la Cámara de Representantes de Puerto Rico, le rindieron un escueto informe negativo, utilizando como único criterio la postura asumida por el Departamento de la Familia. El informe concluye que, a pesar de que esta medida de avanzada persigue un fin loable, las comisiones no recomiendan su aprobación, dado el hecho de que la misma impactaría aún más el frágil cuadro fiscal que enfrenta el gobierno de Puerto Rico. Esta determinación, disfrazada bajo la justificación de salvaguardar las finanzas del estado, es una acción contraria a la protección de los derechos civiles de la personas sin hogar, la responsabilidad del estado para asegurar que toda persona disfrute de un sistema de vida digno y el deber de proveer los servicios esenciales, las oportunidades de rehabilitación y el apoyo necesario para eliminar la desigualdad social y la marginación.

Este informe representa un desacierto a los seis años de encomiable labor en favor de la dignidad humana de un sector de la población que se encuentra rezagado y que carece de una herramienta legal que les revindique ante el sistema de justicia en Puerto Rico. Sin embargo, las comisiones asumieron una postura contradictoria, al séñalar que aunque "están obligadas a endosar toda medida que propenda el mejoramiento de la calidad de vida de los deambulantes... somos de 
la creencia que este no es el mejor mecanismo para lograr dicho fin" (Comisión de Bienestar Social y de Vivienda, 2005, p. 3). Ante esta determinación, debemos concluir que la Comisión erró en su deber ministerial de identificar estrategias creativas a través de un esfuerzo coordinado entre el poder Ejecutivo y Legislativo, las organizaciones de base comunitaria y el sector privado, a los fines de garantizar los derechos de las personas sin hogar (Soto, octubre 2006).

Desde sus orígenes, la profesión de trabajo social ha procurado para que estos derechos no sean privativos de unos pocos, sin embargo, las personas sin hogar continúan siendo excluidas de las oportunidades de progreso que están disponibles para el resto de la población. Ante esta realidad, los profesionales del trabajo social poseen una herramienta para provocar que una iniciativa de esta envergadura sea nuevamente radicada en un esfuerzo para que la misma se convierta en Ley. Esta herramienta es el ejercer su rol como activistas sociales, a través del cual se viabiliza la defensa de un sector de la población que se encuentra desprovisto de sus derechos fundamentales. Tal como establece Ruiz (1997), un activista social es un profesional de ayuda que se involucra en actividades dirigidas a establecer nuevos derechos y prerrogativas para sus clientes, crear conciencia sobre una problemática social y movilizar los recursos disponibles para cambiar las condiciones conducentes a dichos problemas. De esta forma, los trabajadores y trabajadoras sociales podrán contrarrestar el debate que ha caracterizado la discusión de esta medida, en el que las necesidades de las personas sin hogar han sido relegadas a un contexto puramente económico, al ilustrar a nuestros legisladores sobre la reconstrucción de los ideales de justicia social que históricamente ha procurado la profesión.

\section{Política pública y la erradicación del discrimen por condición social: ¿Una ruptura irreparable?}

En un trámite similar, la Comisión de Asuntos Municipales y Financieros así como la de Bienestar Social del Senado de Puerto Rico, no favorecieron el P. del S. 223. Este proyecto estaba dirigido a prohibir que los municipios aprobaran ordenanzas, códigos de orden 
público o cualquier medida que establezca penalidades basadas en la clasificación de ser deambulantes. El mismo surgió en respuesta a las denuncias públicas de que las agencias encargadas de promover la seguridad entre la ciudadanía, estaban siendo utilizadas para arrestar y procesar criminalmente a personas que cometían el "delito" de mendigar en lugares públicos, acción que les criminalizaba utilizando como único criterio su condición social (Colegio de Abogados de Puerto Rico, 2006).

Un proyecto de esta envergadura, no debería confrontar mayores dificultades para ser aprobado en la Legislatura de Puerto Rico, dado a que el mismo es cónsono con las disposiciones de la Sección I del Artículo II de la Constitución del Estado Libre Asociado de Puerto Rico (1952), donde se establece que "no podrá establecerse discrimen alguno por motivo de raza, color, sexo, nacimiento, origen o condición social, ni ideas políticas o religiosas" [énfasis suplido]. Además, esta medida ejemplifica los valores y objetivos que persigue la profesión, por lo que debió haber contado con el respaldo decidido del Colegio de Trabajadores Sociales de Puerto Rico, dado a que sus principios rectores son cónsonos con el código de ética que rige las conductas de sus miembros (1982). Este establece la responsabilidad profesional de prevenir y eliminar el discrimen contra cualquier persona o grupo, particularmente cuando el criterio utilizado es la condición de vida de estas personas. Sin embargo, las Comisiones determinaron, a través de la radicación de un informe negativo, que la medida no debía ser aprobada, utilizando el argumento de que no se deben coartar las funciones de las legislaturas municipales, en su discreción de aprobar aquellas medidas que, a su juicio, redunden en beneficio de la ciudadanía. De esta forma, las disposiciones de la Carta de Derechos que se encuentra consagrada en nuestra Constitución fueron rezagadas a un segundo plano y se le otorgó mayor valía a un asunto administrativo como lo es la autonomía municipal. La profesión perdió una oportunidad invaluable de concienciar a nuestra población de la realidad social que nos rodea, sus repercusiones y de la necesidad de detener una tendencia peligrosa: la criminalización de la pobreza. 


\section{El proceso legislativo y el asunto de la representatividad: El futuro de la prohibición del discrimen por orientación sexual}

Al comienzo de un nuevo siglo, la práctica del trabajo social en Puerto Rico enfrenta el surgimiento de controversias noveles que involucran a clientes homosexuales, lesbianas, transexuales y transgénero. En nuestra jurisdicción, el estado de derecho vigente, carece de una postura concreta sobre los derechos de las personas homosexuales, lo que ha resultado en que sus derechos sean vulnerables, en temas relacionados a la adjudicación de custodia y patria potestad, adopción, provisión de servicios esenciales, concesión de remedios judiciales e igualdad de oportunidades en el empleo, entre otros.

Las diversas formas de discrimen que genera la naturaleza humana, tales como la marginación y coartar las oportunidades de participación de los grupos minoritarios en los procesos sociales, provocó la radicación del Proyecto del Senado 1585, dirigido a promover una sociedad pluralista e inclusiva en la que se prohíba el discrimen por impedimento físico y orientación sexual. A través del mismo, se prohíbe, de manera explícita, negar a una persona servicio e igual trato en lugares públicos y medios de transporte; negarse a vender, arrendar o subarrendar una vivienda y/o negarse a conceder préstamos para la construcción de viviendas, utilizando como criterio la orientación sexual y/o los impedimentos físicos del solicitante.

Empero la pertinencia y necesidad de esta iniciativa, diversos sectores moralistas y religiosos han expresado su rechazo a la medida, utilizando como argumento los valores cristianos prevalecientes en la cultura puertorriqueña. Plantean estos sectores que expresiones oficiales que impliquen una aceptación de las orientaciones sexuales lésbicas, homosexual y transgénero de Puerto Rico traerían como consecuencia una proliferación de estos comportamientos. Inclusive, la presión ejercida por estos sectores fundamentalistas, ha provocado que a un (1) año de su radicación, la Comisión de lo Jurídico, Asuntos Municipales y Financieros no haya programado vistas públicas para analizar los méritos de esta medida. 
Una argumentación que pareciera concordar con esta postura es una reciente decisión del Tribunal Supremo de Puerto Rico en el caso Pueblo de Puerto Rico v. Ruiz Muñiz (2003 TSPR 52), en la que se buscó, sin éxito, equiparar las relaciones heterosexuales y homosexuales en la aplicabilidad de las disposiciones de la ley para la prevención e intervención con la violencia doméstica, popularmente conocida como la Ley 54. No obstante, los jueces asociados Hon. Hernández Denton y Hon. Naveira de Rodón disintieron por escrito de la opinión de la mayoría. Estos argumentaron que nuestro máximo foro judicial debe ser proactivo y emitir una opinión de vanguardia social que le haga justicia a un sector de la población cuya orientación sexual contrasta con el patrón prevaleciente en nuestro país. De esta forma, se quebrantará la concepción de que en la defensa de los derechos de estos sectores, los mismos deben ser tratados como ciudadanos de segunda clase. Este llamado está dirigido a trascender todas las esferas sociales, incluyendo a las clases profesionales que proveen servicios directos a esta población.

El discurso prevaleciente ha sido que las personas homosexuales representan una amenaza a la estabilidad social de nuestro pueblo, lo que demuestra la necesidad de que se adopte una postura institucional del trabajo social como profesión que viabilice un plan de acción para lograr cambiar esta percepción. El mismo deberá estar fundamentado en la evidencia empírica provista por las principales organizaciones psiquiátricas y psicológicas, las cuales han dejado contundentemente establecido que no existe evidencia clínica para sustentar que un estilo de vida homosexual implique la existencia de una patología mental. Por el contrario, la inmensa mayoría de los estudios han concluido que las personas que son homosexuales disfrutan de una buena vida social, afectiva y vocacional (Martínez, 2006).

En el ejercicio de identificar y analizar las condiciones que han provocado la negativa para lograr legislación que ofrezca un frente común en la lucha contra la desigualdad de estos sectores de la población, los profesionales del trabajo social pueden ejercer un rol de intercesores entre el cliente, entiéndase aquellos que son privados 
de los servicios esenciales, al utilizar como criterio sus impedimentos físicos y/o su orientación sexual, y las instituciones proveedoras de servicios, que utilizan tales criterios para obstaculizar su acceso. En el ejercicio de fungir como intercesores, profesionales del trabajo social integran estrategias básicas del modelo de solución de problemas, tales como la definición y evaluación de la problemática, a los fines de desarrollar un plan de acción que viabilice que una medida de esta naturaleza sea convertida en Ley. Esta aspiración podrá ser alcanzada, en una fase inicial, a través de la educación como herramienta de mediación y cambio y, en segunda instancia, a través de la denuncia pública, con la expectativa de que la divulgación incorpore nuevos aliados en la defensa de estos sectores.

\section{El camino hacia la reglamentación de la prostitución y la decriminalización de la adicción en Puerto Rico}

Durante décadas, la política prohibicionista, la cual clasifica como criminal a toda persona que usa, abusa o es dependiente de drogas ilegales (Santiago \& Albizu, 2003), ha sido el modelo de intervención en el manejo de usuarios de sustancias contraladas en Puerto Rico. Los fundamentos y los resultados de este modelo punitivo, incluyendo la criminalización de la drogodependencia y el encarcelamiento masivo como estrategia de cambio social, constituyen un desafío para la práctica del trabajo social en Puerto Rico.

En un esfuerzo de avanzada, el Senado de Puerto Rico aprobó la Resolución 2426, dirigido a que la Comisión de Salud, Bienestar Social y Asuntos de la Mujer realice un estudio sobre el impacto que tendría en Puerto Rico reglamentar la prostitución y legalizar y/o medicar el uso de sustancias controladas. A pesar de que estas han sido controversias centenarias, en la actualidad la Legislatura de Puerto Rico no cuenta con estudios investigativos que capaciten a los legisladores a asumir las posturas apropiadas, descansando en información confiable y desvestida de prejuicios, agendas dirigidas y desinformación. 
Aunque esta medida establece de manera explícita que su fin no es legislar para estos fines, una resolución de investigación es el primer paso para auscultar aquellas áreas que necesitan legislación. Desde esta perspectiva, uno de los grandes retos que enfrenta la realización de un estudio de esta naturaleza, será garantizar que sus conclusiones estén divorciadas del debate prejuiciado y, en su lugar, esté fundamentado en evidencia científica y el peritaje de profesionales en conducta humana, incluyendo a profesionales del trabajo social que ofrecen servicios directos a estas poblaciones. En el inicio del proceso de vistas públicas, los trabajadores y trabajadoras sociales podrán ejercer su rol como educadores (Ruiz, 1997), para promover un paradigma de salud pública que reenfoque la inversión de recursos del estado hacia un sistema integrado de salud. Este nuevo enfoque deberá proveer para la creación de programas de prevención, extender y mejorar los servicios en las comunidades terapéuticas y adoptar un acercamiento salubrista al problema, basado en la decriminalización de la drogodependencia y la reducción de daños en el tratamiento y la rehabilitación de adictos en Puerto Rico (Santiago \& Albizu, 2003). Esto viabilizará que, una vez se finalice la fase investigativa, las recomendaciones que ofrezca la Comisión incluyan un análisis ponderado sobre las implicaciones psicosociales que la reglamentación de estas conductas tendría en la sociedad puertorriqueña.

\section{Política Pública y Trabajo Social: ¿Cómo Podemos Influenciar?}

Los trabajadores y trabajadoras sociales poseemos tres herramientas fundamentales para influenciar el proceso político en Puerto Rico: deponer en las vistas públicas que diariamente realiza la Legislatura de Puerto Rico, promover la radicación de proyectos por petición; y fomentar la denuncia pública para alcanzar los cambios necesarios en beneficio de los sectores desprotegidos de la sociedad puertorriqueña (Soto, noviembre 2006).

El proceso de vistas públicas es una discusión abierta utilizada por la Legislatura de Puerto Rico para recibir el insumo de personas 
interesadas en evaluar los méritos de las medidas que se encuentran ante su consideración. A pesar de que las comisiones legislativas utilizan su discreción en la otorgación de turnos cada vez que una persona, grupo o entidad solicita deponer, por regla general se promueve un proceso inclusivo en el que los planteamientos de las personas interesadas son considerados al momento de determinar si se debe continuar con el trámite ordinario de aprobación de medidas o si el mismo debe ser detenido, a través de la radicación de un informe negativo.

Las entidades profesionales y personas particulares interesadas en colaborar de manera continua en los trabajos que realizan las comisiones permanentes de Cámara y Senado, pueden solicitar formar parte de los recursos que éstas utilizan al momento de analizar los méritos de las medidas que se encuentran ante su consideración. Las Comisiones de Bienestar Social, Asuntos de la Juventud, Asuntos de la Mujer, Vivienda y Salud son una muestra de los comités legislativos que más directamente podrían beneficiarse del acervo de experiencias de la profesión. Los trabajadores y trabajadoras sociales, como grupo, deben tomar la iniciativa de establecer el primer acercamiento a estas comisiones, a fin de establecer el contacto necesario que permita a los legisladores beneficiarse del conocimiento de la profesión.

Una manera formal de lograr tal inserción, a pesar de que no representa un requisito para participar del proceso de vistas públicas, es el mecanismo dispuesto por la Legislatura de Puerto Rico para inscribirse como cabildero, tanto en la Cámara de Representantes (Orden Administrativa 2005-01) como en el Senado de Puerto Rico (Orden Administrativa 05-16). El Colegio de Trabajadores Sociales, las organizaciones estudiantiles de los programas de trabajo social a nivel subgradudado y graduado y profesionales del trabajo social en su carácter individual, son ejemplos de grupos que podrían organizarse con el fin de trasladarse a la legislatura a exponer su posición en representación de aquellos sectores que históricamente han carecido de una adecuada representación legislativa: las personas sin hogar (Ruiz, 2006), los drogodependientes (Negrón, 1998) y aquellos que viven en condiciones de pobreza (Rodríguez, 2001). La empresa 
privada ha sido exitosa en la movilización de recursos hacia la Legislatura de Puerto Rico, para adelantar o detener iniciativas que les afectan como organización. La Asociación de Bancos, la Asociación de Industriales y la Cámara de Comercio son ejemplos de entidades privadas que cuentan con personal capacitado para influenciar el proceso legislativo a través de los cabilderos, a los fines de adelantar los intereses de sus compañías.

Sin embargo, las herramientas principales que deben poseer las entidades interesadas en influenciar el proceso legislativo son voluntad e iniciativa. Las limitaciones económicas no deben representar un obstáculo al momento de un grupo organizarse para inscribirse como cabildero. Los grupos ambientalistas, las organizaciones de padres y madres que defienden los derechos de los niños de educación especial y los padres que educan a sus hijos en el hogar, son ejemplos de grupos que han logrado adelantar la defensa de sus convicciones, sin contar con una fuente de financiamiento que sustente sus funciones. Voluntad e iniciativa, esos son los requisitos primordiales y esenciales de todo movimiento. La profesión del trabajo social las posee, sólo hay que ponerlas en marcha.

\section{Proyectos por petición}

En nuestro sistema constitucional los legisladores son los únicos con la facultad para presentar medidas legislativas. No obstante, existe un mecanismo para que los proyectos sean radiados por petición. Los proyectos por petición son aquellos presentados por solicitud de una persona o grupo y su radicación no implica necesariamente que el legislador que lo presenta endose el mismo.

En ocasiones, los grupos interesados en presentar una medida legislativa por petición, cuentan con personal capacitado para realizar la investigación y la preparación de un anteproyecto de ley, por lo que su intervención está limitada a lograr que un legislador acepte radicar el mismo. En otras ocasiones, estos grupos establecen comunicación con algún legislador, para solicitar la colaboración de su personal en la redacción de un anteproyecto de ley. Sin embargo, 
una vez el proyecto ha sido radicado, las posibilidades de que éste avance a través de las fases ordinarias del proceso legislativo, está atado a la movilización que estos realicen para educar y cabildear, a los fines de conseguir el respaldo necesario que viabilice su aprobación.

Varias organizaciones ambientalistas presentaron el Proyecto de la Cámara 2105, con el fin de proteger la zona que se denomina como el Corredor Ecológico del Noroeste. Estos grupos prepararon la medida, convencieron a representantes de los tres partidos políticos y cabildearon su propuesta intensamente contra los cabilderos de los intereses hoteleros y desarrollistas. El pasado mes de noviembre de 2006, los esfuerzos realizados redundaron en una primera victoria al lograr la aprobación de esta iniciativa ante la Cámara de Representantes. ¿Qué diferencia a los trabajadores y trabajadoras sociales de los grupos ambientalistas? Estos grupos se organizaron y están luchando, ¡hagámoslo nosotros también!

\section{Conclusión}

Las necesidades apremiantes de las clases desprotegidas de la sociedad puertorriqueña, son un reflejo inequívoco de la urgencia para integrar a los profesionales del trabajo social en el ejercicio de desarrollar e influenciar soluciones noveles a los problemas que históricamente han limitado sus oportunidades de progreso. Entre éstos, la negativa para legislar una carta de derechos que proteja su dignidad como seres humanos y que erradique el discrimen hacia un sector que es penalizado por su condición social, representa el rechazo hacia 7,100 personas sin hogar (Departamento de la Familia, 2006) que hoy claman por una justa representación legislativa.

En segunda instancia, los esfuerzos para encausar la gestión pública y comunitaria, a través de la eliminación del discrimen por orientación sexual, representa otra de las grandes encomiendas que enfrentan profesionales del trabajo social al insertarse en el proceso legislativo. Durante la ausencia del Colegio de Trabajadores Sociales como institución y de trabajadores o trabajadoras sociales en su carácter 
individual, el P. del S. 1585 ha sido privado de ser discutido en sus verdaderos méritos en el proceso de vistas públicas.

Empero al fortalecimiento del consenso internacional sobre la necesidad de identificar enfoques integrados y multidimensionales que puedan decriminalizar las adicciones en Puerto Rico, aún persiste el paradigma prohibicionista punitivo para sostener la política restrictiva en el uso de sustancias controladas (Santiago \& Albizu, 2003). Mientras profesionales en conducta humana, tales como las trabajadoras y trabajadores sociales, se inhiban de participar en la formulación de políticas públicas y sociales, va a prevalecer la imposición de medidas represivas que criminalizan a las personas drogodependientes y les limiten sus oportunidades de rehabilitación.

En definitiva, la discusión de estas medidas hace evidente que la participación activa de profesionales del trabajo social en el proceso legislativo debe estar dirigido a que en los próximos años el Colegio de Trabajadores Sociales pueda acoger la recomendación de Guardiola (1997) de establecer una comisión de trabajo social en la Legislatura de Puerto Rico. En esta comisión, trabajadoras y trabajadores sociales puedan desempeñarse como peritos en política pública y legislación. Sin embargo, debemos advertir que, a pesar de que esta recomendación representa un esfuerzo de vanguardia, esta Comisión podría convertirse en un ente inoperante si nuestros legisladores desconocen el peritaje de los profesionales del trabajo social en el desarrollo del proceso legislativo. En su lugar, recomendamos que las comisiones permanentes de Bienestar Social, Asuntos de la Juventud, Asuntos de la Mujer, Vivienda y Salud en Cámara y Senado, sean fortalecidas con la consultaría de trabajadoras y trabajadores sociales que faciliten el proceso de investigación y análisis previo a la presentación de legislación, a los fines de que la misma sea sensible a las necesidades de la sociedad puertorriqueña.

Aún resta un extenso e intenso proceso legislativo que deberá trascender líneas ideológicas, para que las determinaciones finales en asuntos que inciden en los derechos de los sectores vulnerables de la sociedad puertorriqueña, sea un proceso inclusivo dirigido a 
alcanzar una sociedad más justa, fraterna, participativa, solidaria y democrática. En este proceso, los trabajadores y trabajadoras sociales como clase profesional, enfrentarán la gesta histórica de de informarse, organizarse y movilizarse hasta la Legislatura de Puerto Rico para revindicar a las clases desprotegidas a través de la reconstrucción de los postulados de justicia social de nuestros pioneros en la profesión (Burgos, 2001).

\section{Referencias}

Burgos Ortiz, Nilsa M. (2001). Pioneras de la profesión de trabajo social en Puerto Rico. San Juan: Publicaciones Puertorriqueñas: San Juan.

Cámara de Representantes de Puerto Rico (2005). Orden Administrativa 2005-01 [Data File]. San Juan, Puerto Rico:Autor

Colegio de Abogados de Puerto Rico (2006, enero). Resolución Número 39: Para expresar nuestra profunda preocupación sobre la situación de los deambulantes en Puerto Rico y el rechazo a las leyes que permiten el arresto y procesamiento penal de estos por mendigar en lugares públicos. San Juan, Puerto Rico: Autor.

Colegio de Trabajadores Sociales de Puerto Rico (2000). Recomendaciones del Colegio de Trabajadores sociales de Puerto Rico en áreas prioritarias de política pública. San Juan, Puerto Rico: Autor.

Colegio de Trabajadores Sociales de Puerto Rico (1982). El Código de Ética del Profesional de Trabajo Social. San Juan, Puerto Rico: Autor.

Comisión de Asuntos Municipales y Financieros; y de Bienestar Social (2005, noviembre). Informe Negativo Conjunto sobre el Proyecto del Senado 223. San Juan: Senado de Puerto Rico. 
Comisión de Bienestar Social y de Vivienda (2005, noviembre). Informe negativo sobre el Proyecto de la Cámara 1454. San Juan: Cámara de Representantes. 3. Constitución del Estado Libre Asociado de Puerto Rico (1952).Tomo I: Leyes Anotadas de Puerto Rico (LPRA).

Departamento de la Familia (2006). Ponencia sobre la Resolución del Senado 391. Senado de Puerto Rico: San Juan, Puerto Rico: Senado de P.R.

El Pueblo de Puerto Rico v. Ruiz Muñiz (2003, abril). 2003 TSPR 52. San Juan, Puerto Rico. Tribunal Supremo de Puerto Rico

Guardiola, Dagmar \& Serra, José. (1997). El Trabajo Social y los legisladores como formuladotes de política social. Escuela Graduada de Trabajo Social. Río Piedras: Universidad de Puerto Rico.

Hernánez, M. (2006, julio). Proyecto del Senado 1585. San Juan: Senado de Puerto Rico.

Kliksberg, B. \& Rivera, M. (2005). La experiencia de Puerto Rico en la lucha contra la Pobreza. New York: Banco de Desarrollo Económico (UNESCO).

Martínez, A. (2006, noviembre). Homosexualidad y psicología. El Nuevo Día. San Juan, Puerto Rico.

Méndez, S. \& Vizcarrondo, I. (2005, mayo). Proyecto de la Cámara 1454. San Juan: Cámara de Representantes de Puerto Rico. 3-4.

Negrón, G. (1998, mayo). En el limbo la prevención del VIH. El Nuevo Día. Recuperado el 29 de noviembre de 2006 de www.adendi.com. 
Oficina de Servicios Legislativos (2003). La Asamblea Legislativay el Proceso Legislativo. San Juan: Puerto Rico: Autor

Rodríguez, N. (2001). Pobreza y Política Pública en Puerto Rico. Compendio de Lecturas TSOC 3005. Río Piedras: Universidad de Puerto Rico.

Ruiz, G. (2006, enero 12). Grito contra los códigos de orden público. El Nuevo Día. Recuperado el 29 de noviembre de 2006 de www.adendi.com.

Ruiz, Magaly. (1997). Organización de comunidad y política social: Un compendio. San Juan, Puerto Rico: Editorial Edil.

Santiago, Salvador y Albizu, Carmen. (2003, marzo). ¿Guerra contra las drogas o guerra contra la salud? Los retos para la salud pública de la política de las drogas en Puerto Rico. PRHSJ, 22(1).

Santiago, M. (2005, enero). Proyecto del Senado 223. San Juan: Senado de Puerto Rico.Senado de Puerto Rico (2005). Orden Administrativa 05-16: Registro de Cabilderos [Data File]. San Juan: Senado de Puerto Rico.

Soto, D. (2006, octubre). El Proceso Legislativo y su impacto en los Profesionales de ayuda. Conferencia ante estudiantes subgraduados de Trabajo Social del curso TSOC 3005 en la Universidad de Puerto Rico, Recinto de Río Piedras.

Soto, D. (2006, noviembre). ¿Cómo un proyecto se convierte en Ley? Conferencia ante Grupo OIKOS en la Universidad Carlos Albizu, Recinto de San Juan. 
